\title{
Solvent Influence in Obtaining Diverse Coordination Symmetries of Dy(III) Metal Centers in Coordination Polymers: Synthesis, Characterization and Luminescent Properties
}

Nithi Phukan,*a Soumyabrata Goswami, ${ }^{\mathrm{b}}$ Sophia Lipstman ${ }^{\mathrm{a}}$, Israel Goldberg, ${ }^{\mathrm{a}}$ and Bharat Kumar Tripuramallu*c

${ }^{a}$ School of Chemistry, Sackler Faculty of Exact Sciences, Tel-Aviv University, Ramat-Aviv, 69978 Tel-Aviv, Israel

${ }^{b}$ Department of Chemistry, Amity Institute of Applied Sciences (AIAS), Amity University Kolkata, Major Arterial Road, Action Area II, Rajarhat, Newtown, Kolkata 700156, India

${ }^{c}$ Chemistry Division, Department of Sciences and Humanities, Vignan Foundation for Science Technology and Research, Vadlamudi, Guntur 522213, Andhra Pradesh, India

\section{Contents:}

Section S1: Synthetic scheme and characterization of the linker $\mathrm{H}_{2} \mathrm{~L}$

Section S2: Asymmetric units of compounds

Section S3: IR spectra of compounds

Section S4: PXRD studies

Section S5: Thermogravimetric curves of compounds

Section S6: Emission studies of the linker $\mathrm{H}_{2} \mathrm{~L}$

Section S7: Details of SHAPE analyses

Section S8: Additional Structural details of the compounds 
Section-S1. Synthesis and characterization of the linker $\mathrm{H}_{2} \mathrm{~L}$

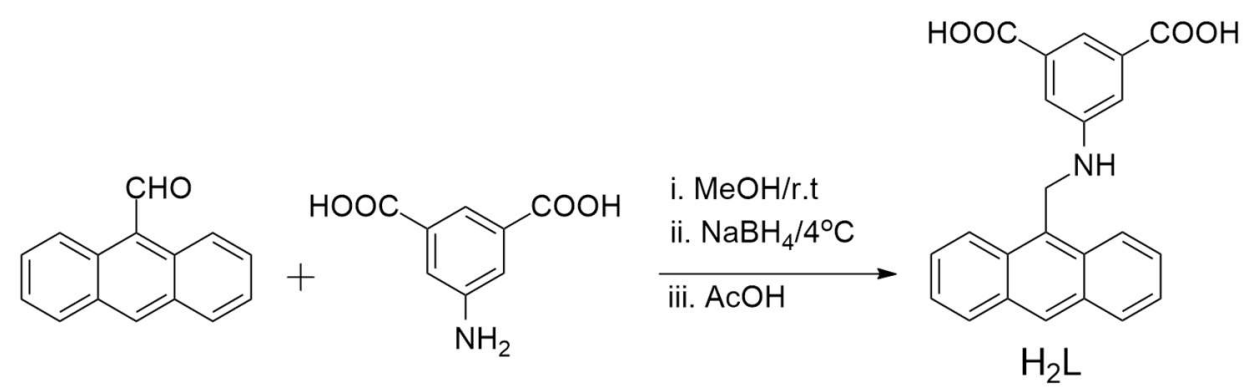

Scheme S1: Synthetic route of Ligand $\mathrm{H}_{2} \mathrm{~L}$

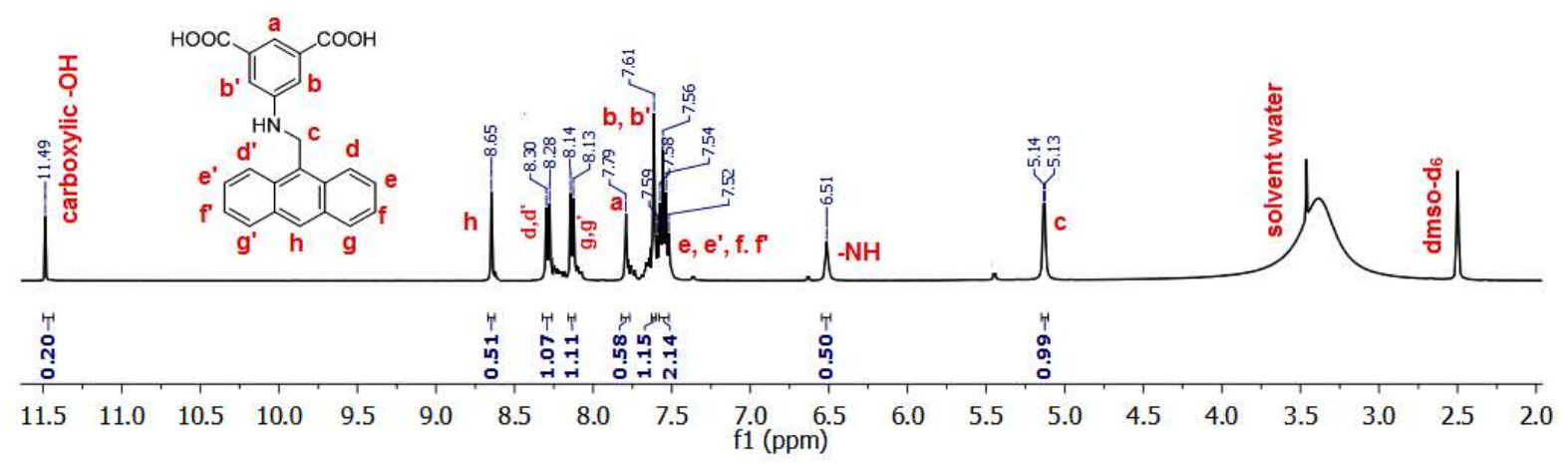

Figure S1. ${ }^{1} \mathrm{H}-\mathrm{NMR}$ (dmso-d $\mathrm{d}_{6}, 400 \mathrm{MHz}$ ) spectra of ligand $\mathrm{H}_{2} \mathrm{~L}$.

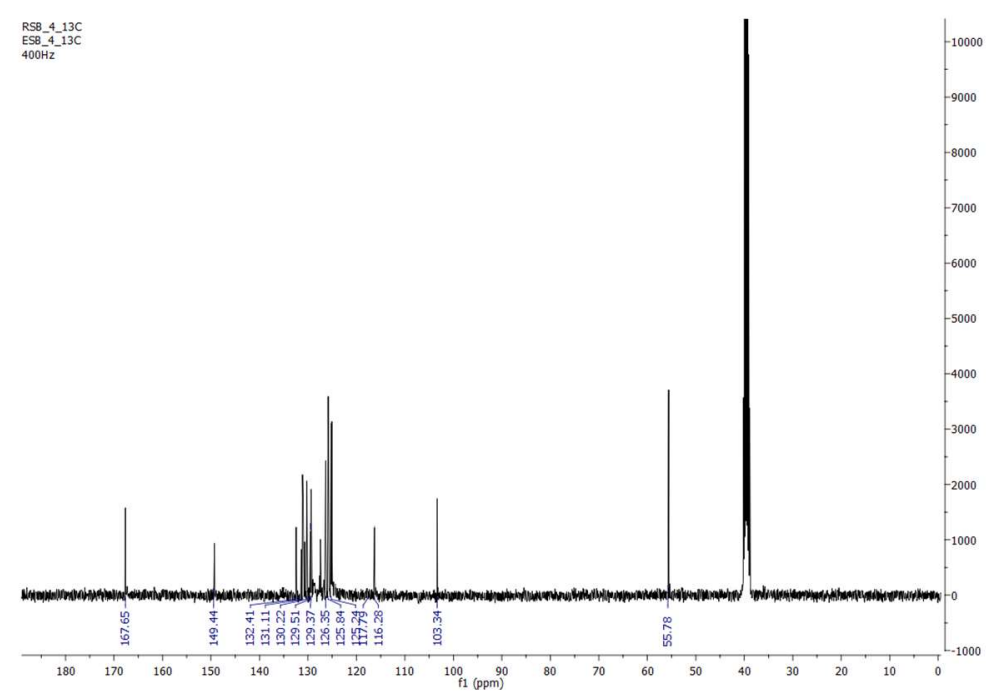

Figure S2. ${ }^{13} \mathrm{C}-\mathrm{NMR}\left(\mathrm{dmso}_{\mathrm{d}}, 100 \mathrm{MHz}\right)$ of ligand $\mathrm{H}_{2} \mathrm{~L}$ 


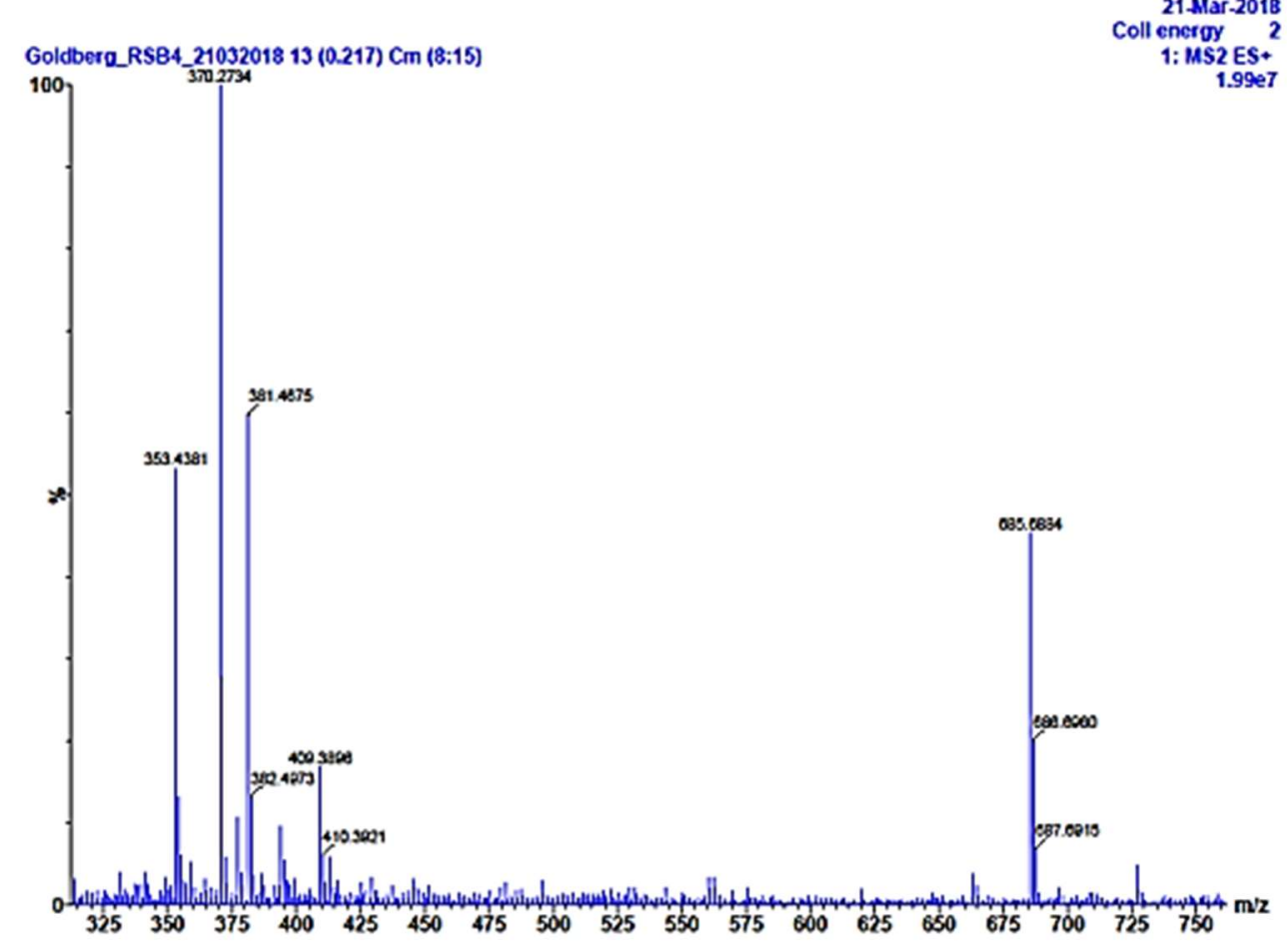

Figure S3. ESI-MS spectra of ligand $\mathrm{H}_{2} \mathrm{~L}$

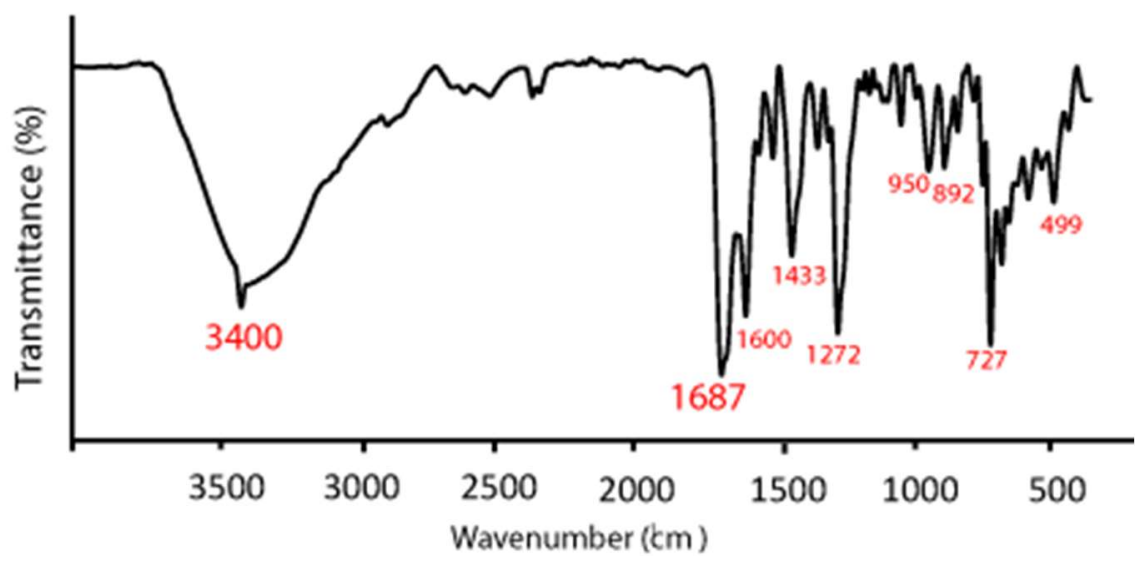

Figure S4. IR spectrum of ligand $\mathrm{H}_{2} \mathrm{~L}$ 
Section S2: Asymmetric units of the compounds 1-6.

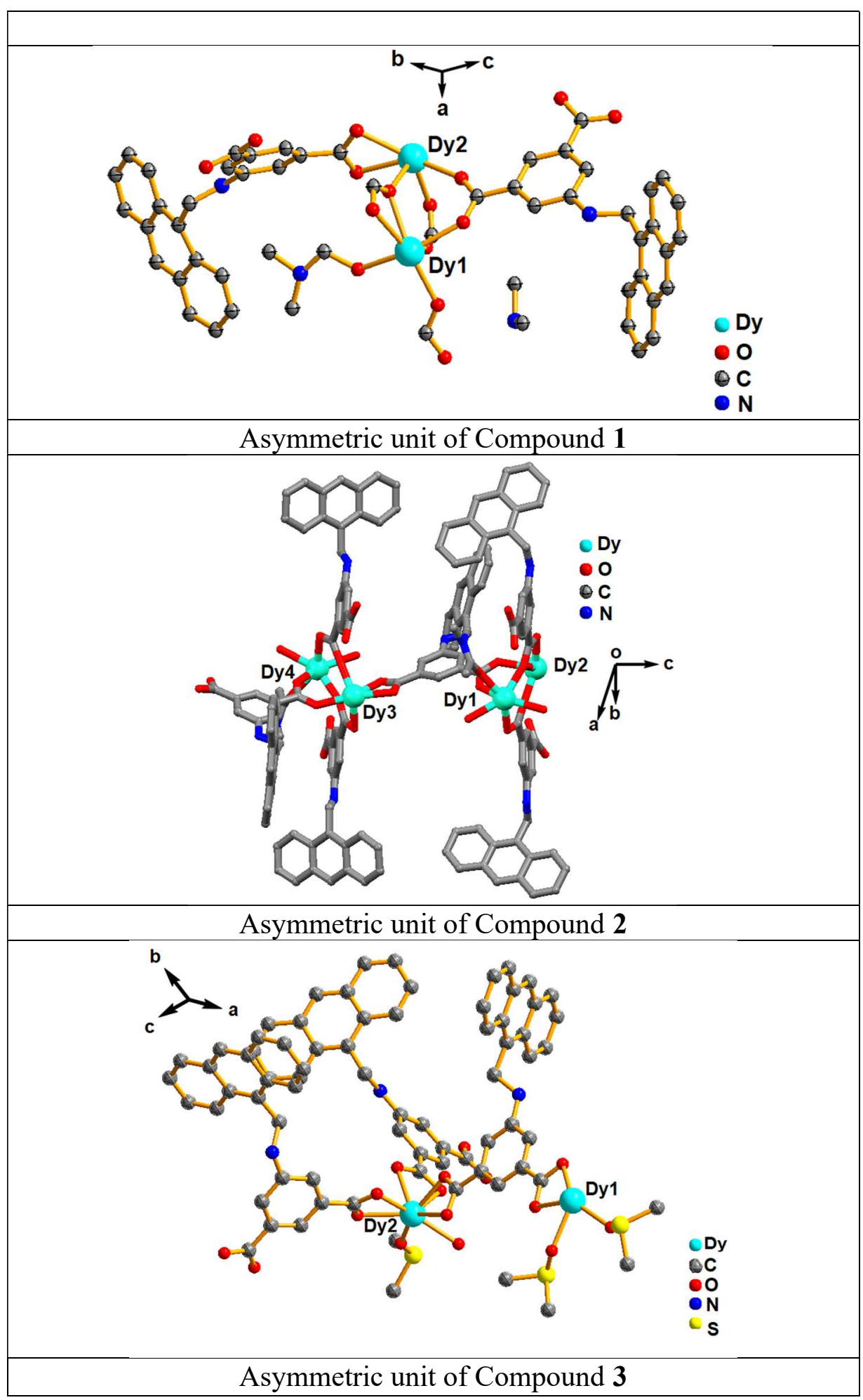




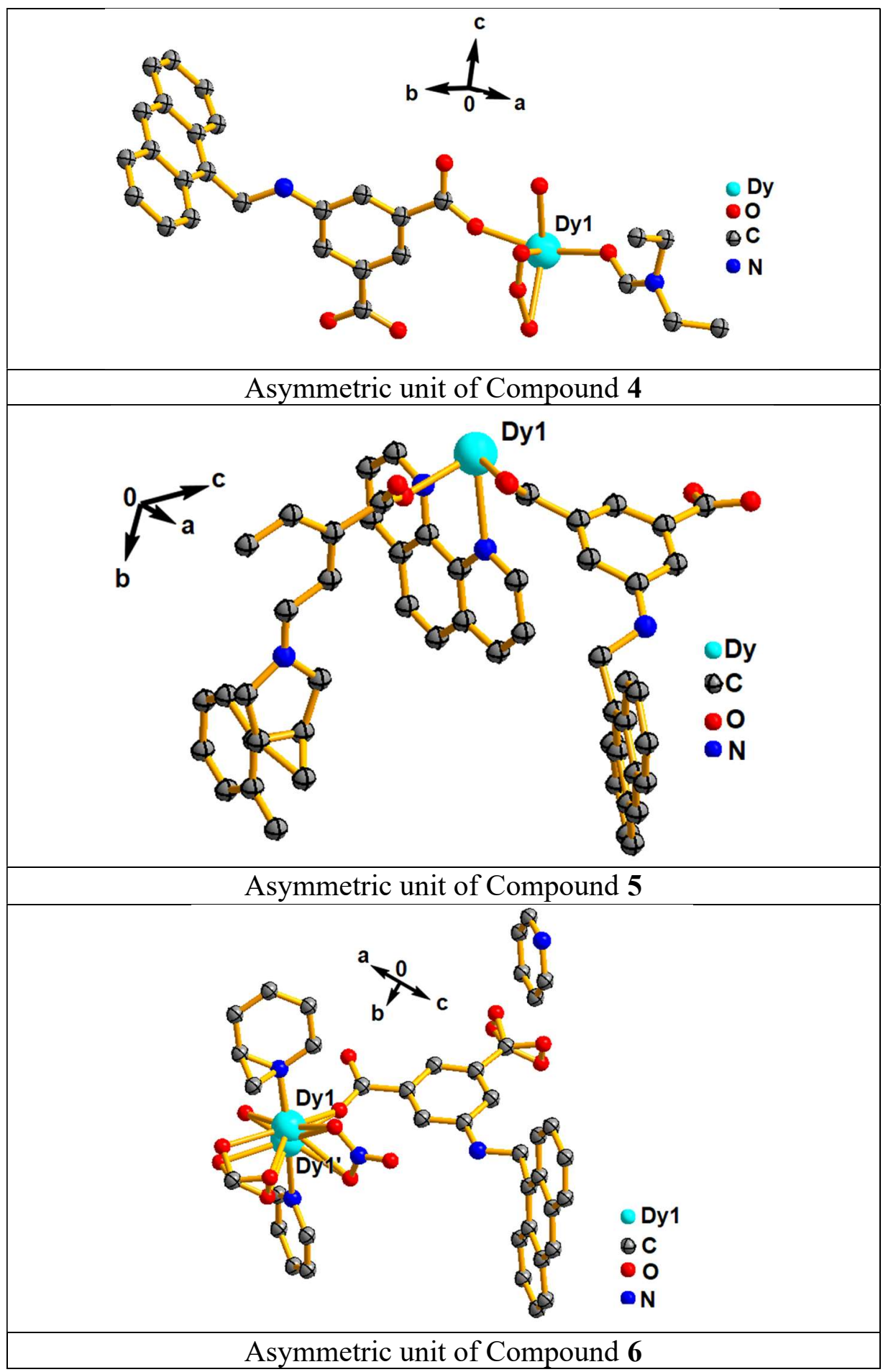


Section S3: IR spectra of compounds 1-6.

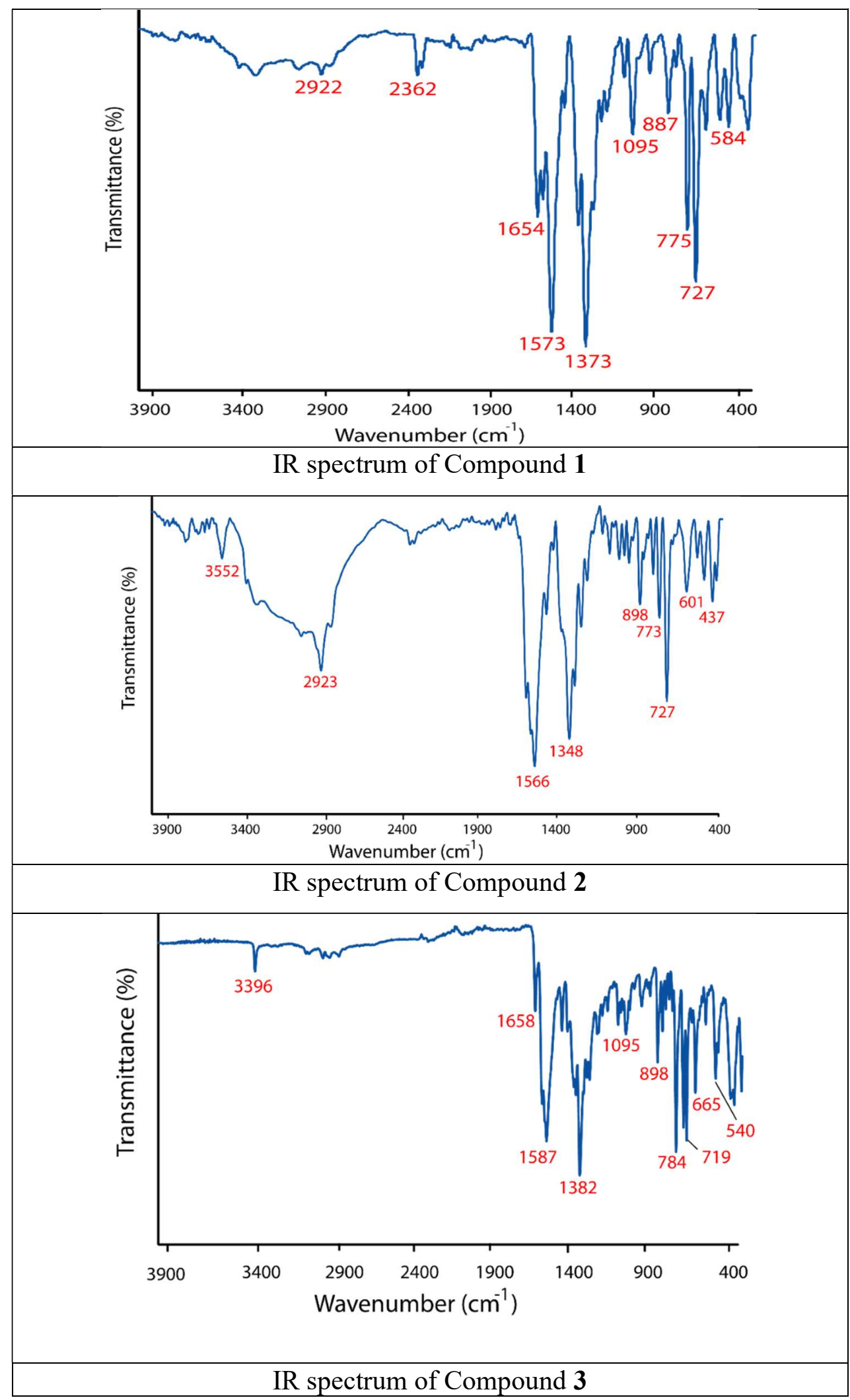




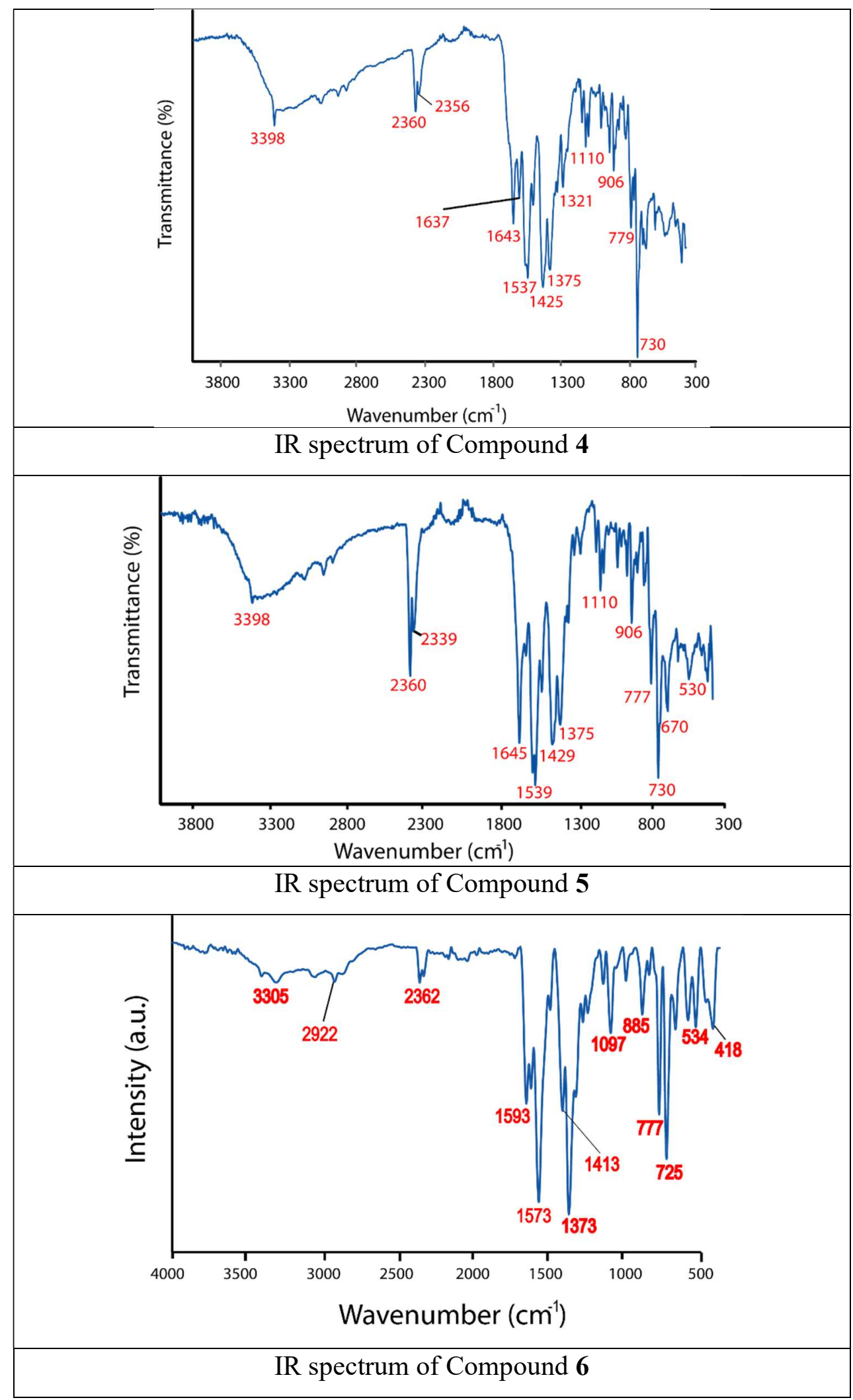


Section S4: PXRD curves of compounds 1-6.

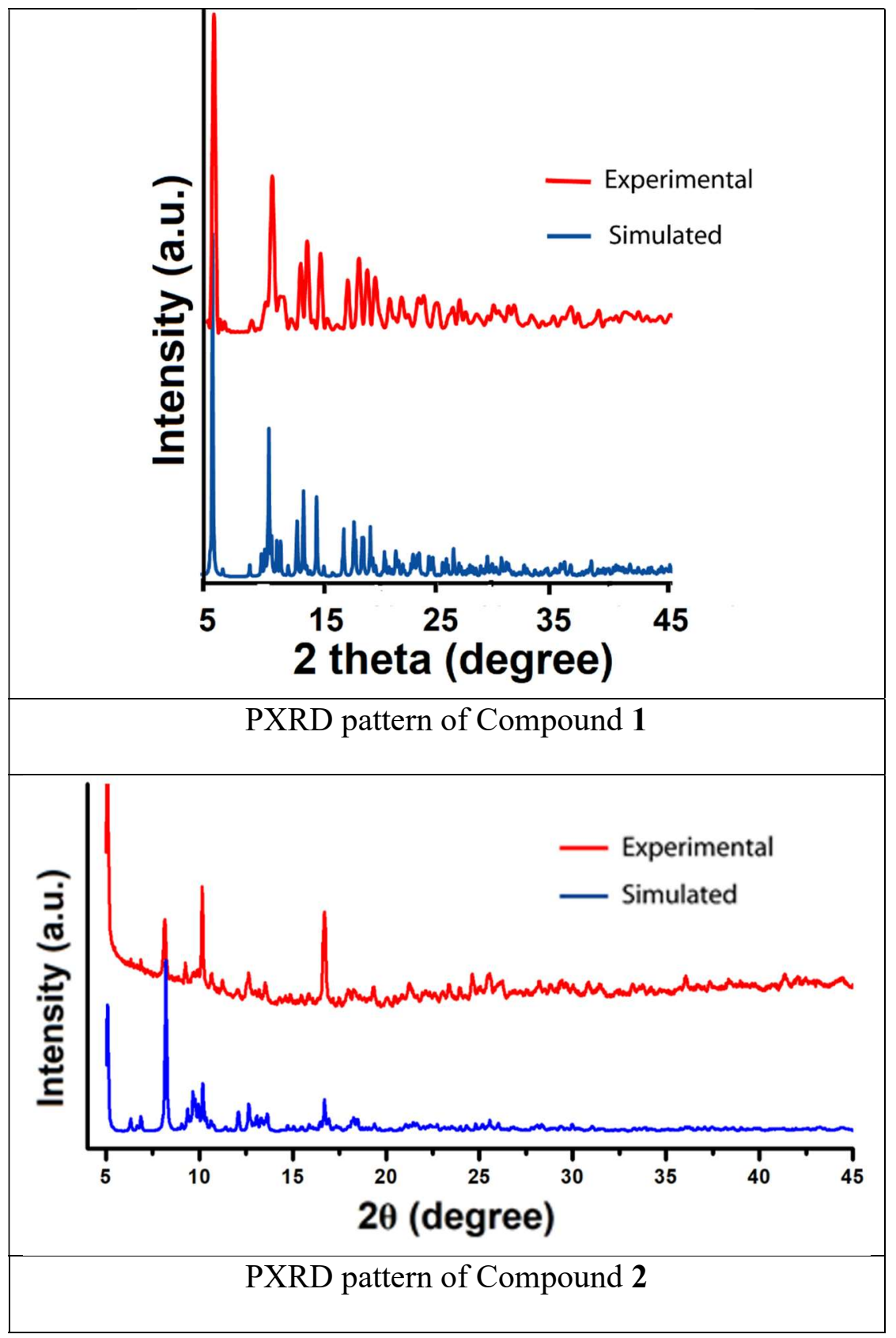




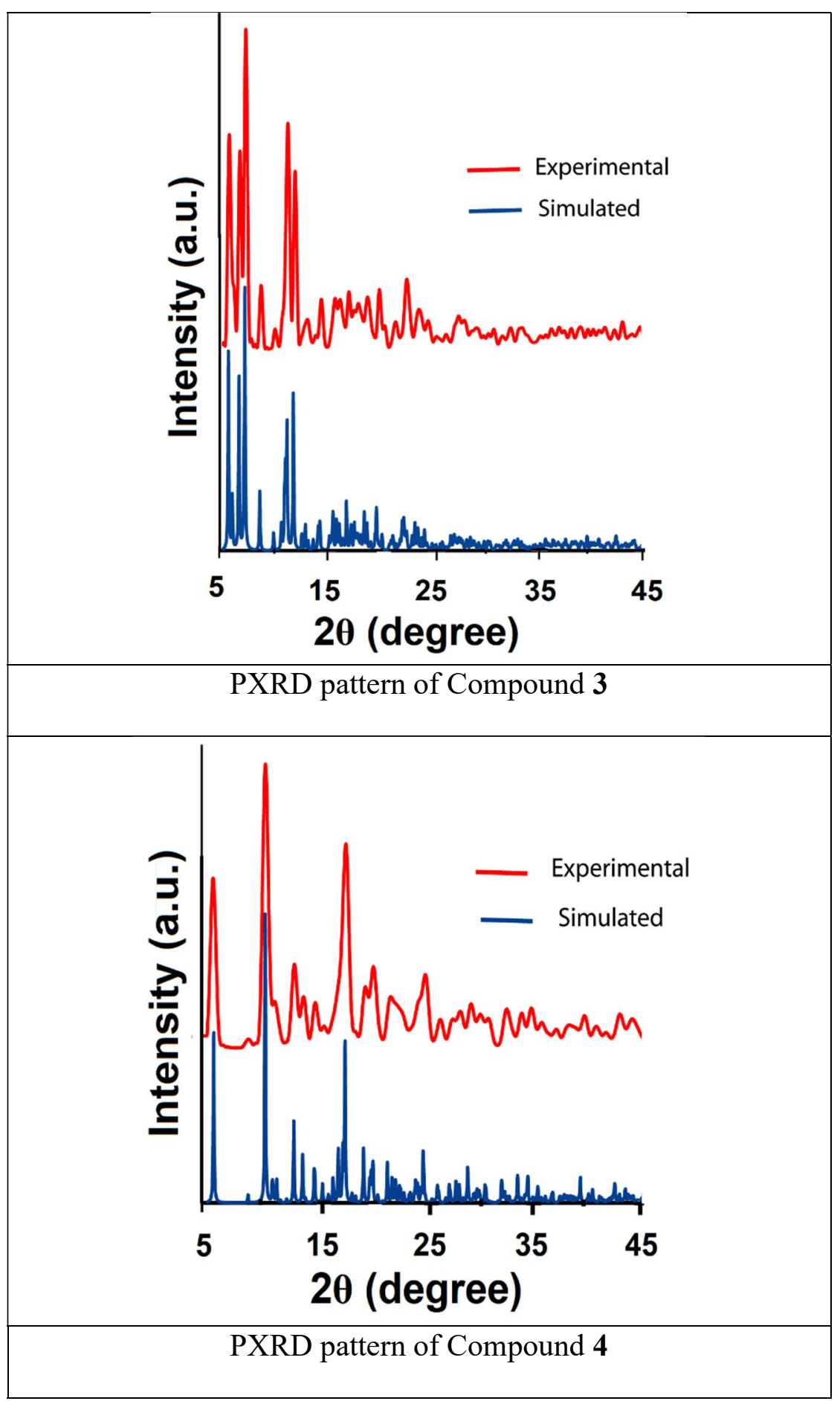




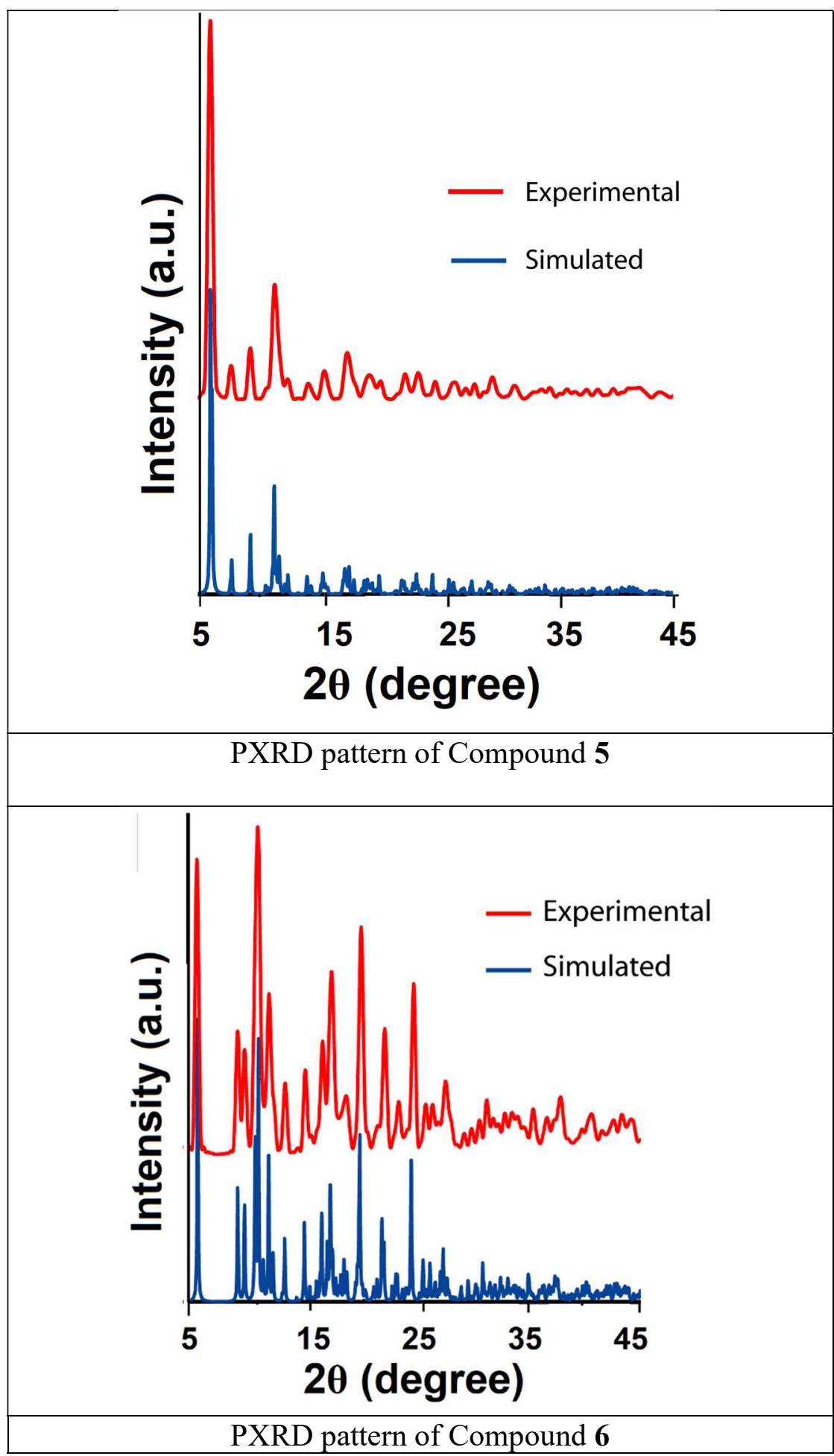


Section S5: Thermogravimetric curves of compounds 1-6.

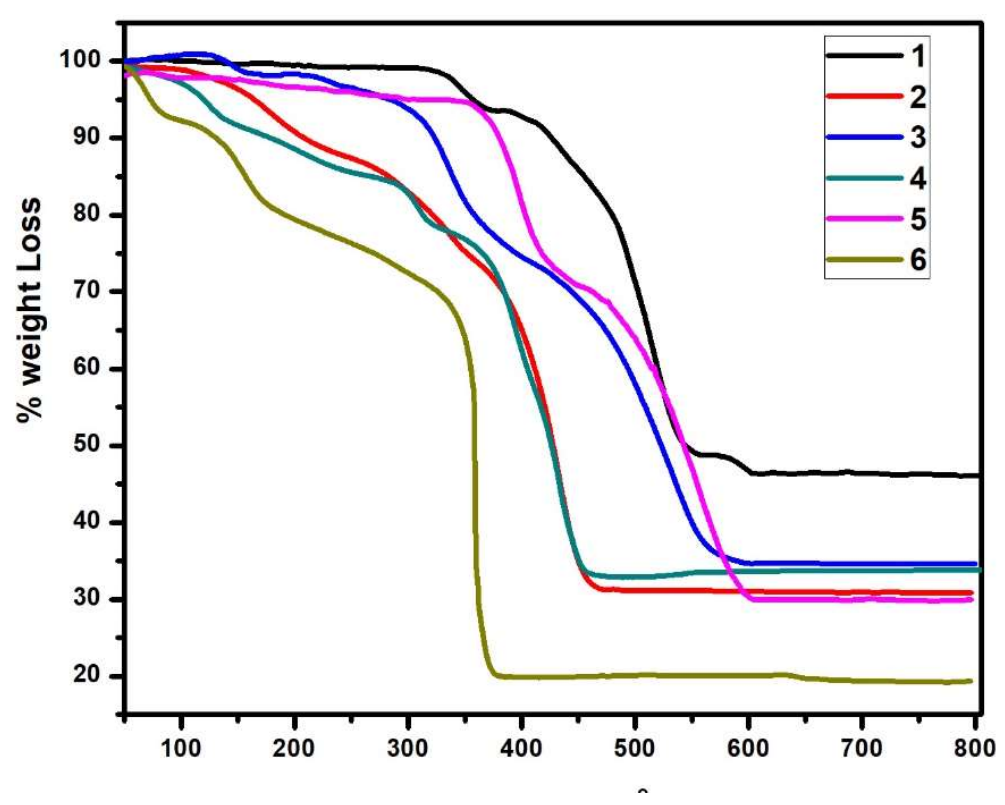

Figure S6. Thermal decomposition profiles of compounds 1-6.

Section S6: Emission studies

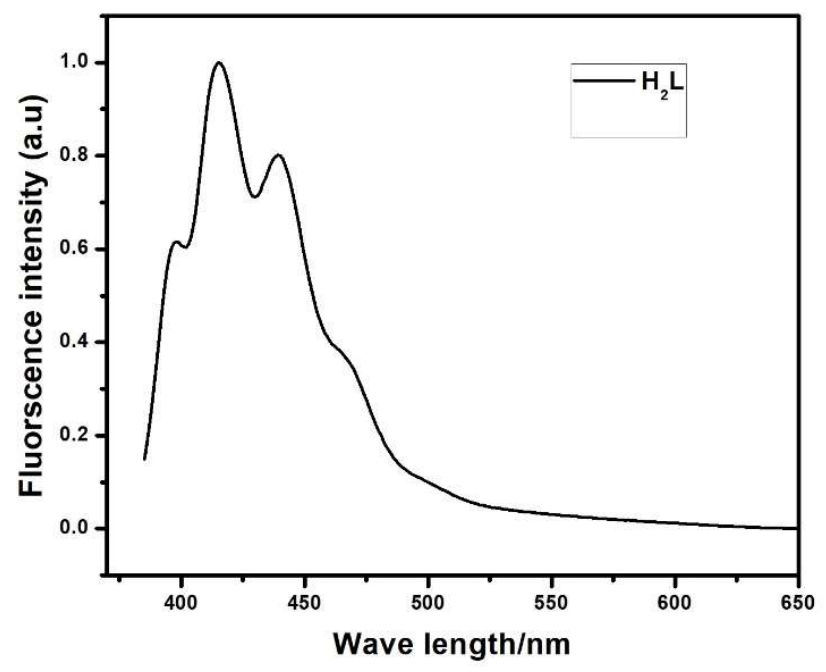

Figure S7: Solution state emission spectra of the linker $\mathrm{H}_{2} \mathrm{~L}$ (upon excitation at $368 \mathrm{~nm}$ ). 
Section S7: Details of the SHAPE analyses

Table S1. Summary of SHAPE analysis for Dy (III) centers in complexes 1 - 6.

Various probable shapes / geometries for coordination number 6:

\begin{tabular}{|c|c|c|l|c|}
\hline Vertices & Code & Label & \multicolumn{1}{|c|}{ Shape } & Symmetry \\
\hline \multirow{6}{*}{} & 1 & HP-6 & Hexagon & $D_{6 h}$ \\
\cline { 2 - 5 } & 2 & PPY-6 & Pentagonal pyramid & $C_{5 v}$ \\
\cline { 2 - 5 } 6 & 3 & OC-6 & Octahedron & $O_{h}$ \\
\cline { 2 - 5 } & 4 & TPR-6 & Trigonal prism & $D_{3 h}$ \\
\hline & 5 & JPPY-5 & $\begin{array}{l}\text { Johnson pentagonal pyramid } \\
(\mathrm{J} 2)\end{array}$ & $C_{5 v}$ \\
\hline
\end{tabular}

Various probable shapes / geometries for coordination number 8 :

\begin{tabular}{|c|c|c|c|c|}
\hline Vertice & Code & Label & Shape & Symmetry \\
\hline \multirow{13}{*}{8} & 1 & OP-8 & Octagon & $D_{8 h}$ \\
\hline & 2 & HPY -8 & Heptagonal pyramid & $C_{7 v}$ \\
\hline & 3 & HBPY -8 & Hexagonal bipyramid & $D_{6 h}$ \\
\hline & 4 & CU-8 & Cube & $O_{h}$ \\
\hline & 5 & SAPR-8 & Square antiprism & $D_{4 d}$ \\
\hline & 6 & TDD-8 & Triangular dodecahedron & $D_{2 d}$ \\
\hline & 7 & JGBF-8 & Johnson - Gyrobifastigium (J26) & $D_{2 d}$ \\
\hline & 8 & JETBPY-8 & $\begin{array}{l}\text { Johnson - Elongated triangular } \\
\text { bipyramid (J14) }\end{array}$ & $D_{3 h}$ \\
\hline & 9 & JBTP-8 & $\begin{array}{l}\text { Johnson - Biaugmented trigonal } \\
\text { prism (J50) }\end{array}$ & $C_{2 v}$ \\
\hline & 10 & BTPR-8 & Biaugmented trigonal prism & $C_{2 v}$ \\
\hline & 11 & JSD-8 & Snub disphenoid (J84) & $D_{2 d}$ \\
\hline & 12 & TT-8 & Triakis tetrahedron & $T_{d}$ \\
\hline & 13 & ETBPY-8 & Elongated trigonal bipyramid & $D_{3 h}$ \\
\hline
\end{tabular}


Various probable shapes / geometries for coordination number 9:

\begin{tabular}{|c|c|c|c|c|}
\hline Vertices & Code & Label & Shape & Symmetry \\
\hline \multirow{13}{*}{9} & 1 & EP-9 & Enneagon & $\mathrm{D}_{9 h}$ \\
\hline & 2 & OPY-9 & Octagonal pyramid & $\mathrm{C}_{8 v}$ \\
\hline & 3 & HBPY-9 & Heptagonal bipyramid & $\mathrm{D}_{7 h}$ \\
\hline & 4 & JTC-9 & $\begin{array}{l}\text { Triangular cupola }(\mathrm{J} 3)= \\
\text { trivacant cuboctahedron }\end{array}$ & $\mathrm{C}_{3 v}$ \\
\hline & 5 & JCCU-9 & $\begin{array}{l}\text { Capped cube (Elongated square } \\
\text { pyramid, J8) }\end{array}$ & $\mathrm{C}_{4 v}$ \\
\hline & 6 & CCU-9 & Capped cube & $\mathrm{C}_{4 v}$ \\
\hline & 7 & JCSAPR-9 & $\begin{array}{l}\text { Capped sq. antiprism } \\
\text { (Gyroelongated square pyramid } \\
\mathrm{J} 10 \text { ) }\end{array}$ & $\mathrm{C}_{4 v}$ \\
\hline & 8 & CSAPR-9 & $\begin{array}{l}\text { Spherical Capped square } \\
\text { antiprism }\end{array}$ & $\mathrm{C}_{4 v}$ \\
\hline & 9 & JTCTPR-9 & Tricapped trigonal prism (J51) & $\mathrm{D}_{3 h}$ \\
\hline & 10 & TCTPR-9 & $\begin{array}{l}\text { Spherical Tricapped trigonal } \\
\text { prism }\end{array}$ & $\mathrm{D}_{3 h}$ \\
\hline & 11 & JTDIC-9 & Tridiminished icosahedron (J63) & $\mathrm{C}_{3 v}$ \\
\hline & 12 & HН-9 & Hula-hoop & $\mathrm{C}_{2 v}$ \\
\hline & 13 & MFF-9 & Muffin & $\mathrm{C}_{s}$ \\
\hline
\end{tabular}

\section{Complex 1 (Dy1)}

$\begin{array}{llllllll}\text { Structure }[\text { ML8 }] & \text { OP-8 } & \text { HPY-8 } & \text { HBPY-8 } & \text { CU-8 } & \text { SAPR-8 } & \text { TDD-8 } & \text { JGBF-8 }\end{array}$ $\begin{array}{lllll}\text { JETBPY-8 JBTPR-8 } & \text { BTPR-8 } & \text { JSD-8 } & \text { TT-8 } & \text { ETBPY-8 }\end{array}$
Dy,
$32.185, \quad 23.633, \quad 13.240, \quad 12.040$,
$4.127, \quad 2.439$,
$11.910, \quad 27.140$

$3.492, \quad 2.969, \quad 3.673, \quad 12.776, \quad 23.702$

(Dy2)

$\begin{array}{llllllll}\text { Structure }[\text { ML8 }] & \text { OP-8 } & \text { HPY-8 } & \text { HBPY-8 } & \text { CU-8 } & \text { SAPR-8 } & \text { TDD-8 } & \text { JGBF-8 }\end{array}$ $\begin{array}{lllll}\text { JETBPY-8 JBTPR-8 } & \text { BTPR-8 } & \text { JSD-8 } & \text { TT-8 } & \text { ETBPY-8 }\end{array}$

Dy $\quad, \quad 31.602, \quad 21.463, \quad 13.363, \quad 12.514, \quad 3.671, \quad 1.964, \quad 12.138, \quad 28.513$, $3.642, \quad 2.803, \quad 3.617, \quad 13.286, \quad 24.382$ 


\section{Complex 2}

Dy1

$\begin{array}{llllllll}\text { Structure }[\text { ML8 }] & \text { OP-8 } & \text { HPY-8 } & \text { HBPY-8 } & \text { CU-8 } & \text { SAPR-8 } & \text { TDD-8 } & \text { JGBF-8 }\end{array}$ $\begin{array}{lllll}\text { JETBPY-8 JBTPR-8 } & \text { BTPR-8 } & \text { JSD-8 } & \text { TT-8 } & \text { ETBPY-8 }\end{array}$

Dy $\quad, \quad 30.320,22.137, \quad 14.614, \quad 11.599, \quad 1.611, \quad 2.096, \quad 12.488, \quad 27.033$, $2.245, \quad 1.410, \quad 4.233, \quad 12.172, \quad 22.602$

Dy2

$\begin{array}{llllllll}\text { Structure }[\text { ML8 }] & \text { OP-8 } & \text { HPY-8 } & \text { HBPY-8 } & \text { CU-8 } & \text { SAPR-8 } & \text { TDD-8 } & \text { JGBF-8 }\end{array}$ $\begin{array}{lllll}\text { JETBPY-8 JBTPR-8 } & \text { BTPR-8 } & \text { JSD-8 } & \text { TT-8 } & \text { ETBPY-8 }\end{array}$

Dy $\quad, \quad 31.470, \quad 18.716, \quad 15.011, \quad 12.547, \quad 5.201, \quad 3.998, \quad 11.367, \quad 24.878$, $3.558, \quad 3.343, \quad 4.741, \quad 12.700, \quad 23.088$

Dy3

$\begin{array}{llllllll}\text { Structure }[\text { ML8 }] & \text { OP-8 } & \text { HPY-8 } & \text { HBPY-8 } & \text { CU-8 } & \text { SAPR-8 } & \text { TDD-8 } & \text { JGBF-8 }\end{array}$

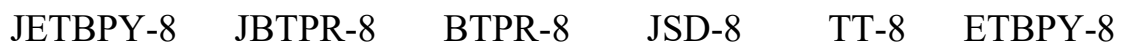

Dy $\quad$ ～29.895, 22.564, 15.589, 11.200, 1.269, 2.381, 13.816, 26.536, $2.901, \quad 1.961, \quad 4.741, \quad 11.970, \quad 22.375$

Dy4

$\begin{array}{llllllll}\text { Structure }[\text { ML8 }] & \text { OP-8 } & \text { HPY-8 } & \text { HBPY-8 } & \text { CU-8 } & \text { SAPR-8 } & \text { TDD-8 } & \text { JGBF-8 }\end{array}$

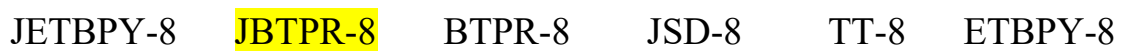

Dy $\quad 31.098, \quad 18.987, \quad 14.862, \quad 12.803, \quad 5.849, \quad 4.408, \quad 10.968, \quad 23.274$, $4.075, \quad 4.180, \quad 4.687, \quad 12.931, \quad 22.070$

\section{Complex 3}

Dy1

Structure [ML9] $\quad$ EP-9 $\quad$ OPY-9 $\quad$ HBPY-9 $\quad$ JTC-9 $\quad$ JCCU-9 $\quad$ CCU-9 JCSAPR-9 CSAPR-9 JTCTPR-9 TCTPR-9 JTDIC-9 HH-9 $\quad$ MFF-9 


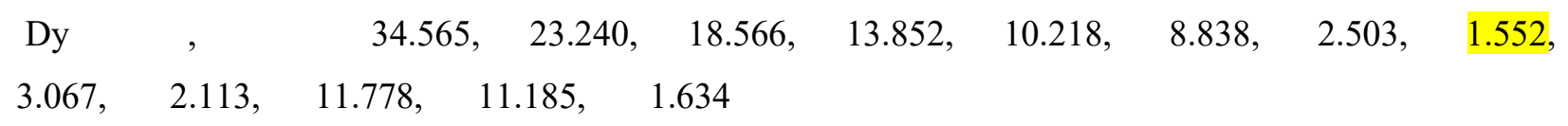

Dy2

Structure [ML9] $\quad$ EP-9 $\quad$ OPY-9 HBPY-9 JTC-9 JCCU-9 $\quad$ CCU-9 JCSAPR-9 CSAPR-9 JTCTPR-9 TCTPR-9 JTDIC-9 HH-9 MFF-9

Dy $\quad$ 32.728, $\quad 24.084, \quad 17.853, \quad 12.595, \quad 9.418, \quad 9.187, \quad 2.897, \quad 2.539$, $2.842, \quad 2.715, \quad 11.564, \quad 11.937, \quad 2.522$

\section{Complex 4}

Dy1

$\begin{array}{llllll}\text { Structure [ML6 ] } & \text { HP-6 } & \text { PPY-6 } & \text { OC-6 } & \text { TPR-6 } & \text { JPPY-6 }\end{array}$

Dy $\quad$ 27.708, $\quad 1.172, \quad 27.771, \quad 14.273, \quad 2.755$

\section{Complex 5}

Dy1

$\begin{array}{llllllll}\text { Structure }[\mathbf{M L 8}] & \text { OP-8 } & \text { HPY-8 } & \text { HBPY-8 } & \text { CU-8 } & \text { SAPR-8 } & \text { TDD-8 } & \text { JGBF-8 }\end{array}$ $\begin{array}{lllll}\text { JETBPY-8 JBTPR-8 } & \text { BTPR-8 } & \text { JSD-8 } & \text { TT-8 } & \text { ETBPY-8 }\end{array}$

Dy $\quad$ 31.196, 20.444, 14.696, 9.631, $1.227, \quad 2.556, \quad 15.955, \quad 27.441$, $3.102, \quad 2.084, \quad 5.487, \quad 10.363, \quad 22.062$

\section{Complex 6}

Dy1

$\begin{array}{llllllll}\text { Structure }[\text { ML8 }] & \text { OP-8 } & \text { HPY-8 } & \text { HBPY-8 } & \text { CU-8 } & \text { SAPR-8 } & \text { TDD-8 } & \text { JGBF-8 }\end{array}$ $\begin{array}{lllll}\text { JETBPY-8 JBTPR-8 } & \text { BTPR-8 } & \text { JSD-8 } & \text { TT-8 } & \text { ETBPY-8 }\end{array}$

Dy $\quad$ 32.799, 20.450, $\quad 13.787, \quad 11.877, \quad 6.397, \quad 3.887, \quad 14.096, \quad 26.992$, $5.175, \quad 4.485, \quad 6.838, \quad 12.605, \quad 23.411$ 
Section S8: Additional Structural details of the compounds 1-6.

\begin{tabular}{|c|c|c|c|c|c|}
\hline Compound & $\begin{array}{l}\text { Squee } \\
\text { zed } \\
\text { electro } \\
\text { ns }\end{array}$ & $\mathbf{Z}$ & $\begin{array}{l}\text { Squeezed } \\
\text { electrons per } \\
\text { Molecular } \\
\text { formula }\end{array}$ & $\begin{array}{l}\text { Estimated } \\
\text { Solvents* }\end{array}$ & Plausible Molecular formula \\
\hline 1 & 15 & 2 & 8 & $1 \mathrm{H}_{2} \mathrm{O}$ & {$\left[\mathrm{Dy}_{2}(\mathrm{~L})_{2}(\mathrm{HCOO})_{3}(\mathrm{DMF}) \cdot \mathrm{Me}_{2} \mathrm{NH}_{2} \cdot \mathrm{H}_{2} \mathrm{O}\right]_{\mathrm{n}}$} \\
\hline 2 & 452 & 2 & 226 & $\begin{array}{l}4 \text { DMA and } \\
4 \mathrm{H}_{2} \mathrm{O}\end{array}$ & {$\left[\mathrm{Dy}_{4}(\mathrm{~L})_{6}(\mathrm{DMA})_{2}\left(\mathrm{H}_{2} \mathrm{O}\right)_{4} \cdot 4 \mathrm{DMA} \cdot 4 \mathrm{H}_{2} \mathrm{O}\right]_{\mathrm{n}}$} \\
\hline 3 & 200 & 2 & 100 & $\begin{array}{c}2 \mathrm{DMSO}+2 \\
\mathrm{H} 2 \mathrm{O}\end{array}$ & {$\left[\mathrm{Dy}_{2}(\mathrm{~L})_{3}(\mathrm{DMSO})_{3}\left(\mathrm{H}_{2} \mathrm{O}\right)_{2} \cdot 2 \mathrm{DMSO} \cdot 2 \mathrm{H}_{2} \mathrm{O}\right]_{\mathrm{n}}$} \\
\hline 4 & \multicolumn{4}{|c|}{ Squeezing not done } & {$\left[\{\mathrm{Dy}(\mathrm{L})(\mathrm{DEF})\}_{2}\left(\mu_{2}-\mathrm{OH}\right)\right]_{\mathrm{n}}$} \\
\hline 5 & 424 & 4 & 106 & $1 \mathrm{DMA} \cdot 7 \mathrm{H}_{2} \mathrm{O}$ & {$\left[\mathrm{Dy}_{2}(\mathrm{~L})_{3}(\mathrm{phen})_{2} \cdot \mathrm{DMA} \cdot 7 \mathrm{H}_{2} \mathrm{O}\right]_{\mathrm{n}}$} \\
\hline 6 & \multicolumn{4}{|c|}{ Squeezing not done } & {$\left[\mathrm{Dy}(\mathrm{L})\left(\mathrm{NO}_{3}\right)(\mathrm{py})_{2} \cdot \mathrm{py}\right]_{\mathrm{n}}$} \\
\hline
\end{tabular}

* solvent molecules are estimated based on TGA curves

Table S2. Table displaying the coordination characteristics of the compounds 1-6

\begin{tabular}{|c|c|c|c|c|c|c|}
\hline C. No & $\begin{array}{l}\text { Metal } \\
\text { Centre }\end{array}$ & $\begin{array}{l}\text { No. of } \\
\text { linkers in } \\
\text { coordination } \\
\text { sphere }\end{array}$ & $\begin{array}{l}\text { Number of } \\
\text { solvent } \\
\text { molecules }\end{array}$ & Coordination sphere & $\begin{array}{l}\text { Coordination } \\
\text { Number }\end{array}$ & $\begin{array}{l}\text { Dimensionalit } \\
y \text { of the } \\
\text { polymer }\end{array}$ \\
\hline 1 & Dy1 & 2 & $\begin{array}{l}\text { DMF-1 } \\
\text { Formate-3 }\end{array}$ & $\begin{array}{c}\text { Triangular } \\
\text { dodecahedron }\end{array}$ & $8\left(\mathrm{D}_{2 \mathrm{~d}}\right)$ & \multirow[t]{2}{*}{$2 \mathrm{D}$} \\
\hline & Dy2 & 3 & Formate -2 & $\begin{array}{c}\text { Triangular } \\
\text { dodecahedron }\end{array}$ & $8\left(\mathrm{D}_{2 \mathrm{~d}}\right)$ & \\
\hline \multirow[t]{4}{*}{2} & Dy1 & 4 & $\begin{array}{l}\text { DMA-1 } \\
\text { Water-2 }\end{array}$ & $\begin{array}{l}\text { Biaugmented } \\
\text { trigonal prism }\end{array}$ & $8\left(\mathrm{C}_{2 \mathrm{v}}\right)$ & \multirow{4}{*}{$2 \mathrm{D}$} \\
\hline & Dy2 & 5 & & $\begin{array}{l}\text { Biaugmented } \\
\text { trigonal prism }\end{array}$ & $8\left(\mathrm{C}_{2 \mathrm{v}}\right)$ & \\
\hline & Dy3 & 4 & $\begin{array}{l}\text { DMA-1 } \\
\text { Water-2 }\end{array}$ & Square antiprism & $8\left(D_{4 d}\right)$ & \\
\hline & Dy4 & 5 & & $\begin{array}{c}\text { Johnson } \\
\text { Biaugmented } \\
\text { trigonal prism }\end{array}$ & $8\left(C_{2 v}\right)$ & \\
\hline \multirow[t]{2}{*}{3} & Dy1 & 3 & $\begin{array}{l}\text { DMSO-2 } \\
\mathrm{H}_{2} \mathrm{O}-1\end{array}$ & $\begin{array}{l}\text { Spherical } \\
\text { Capped square } \\
\text { antiprism }\end{array}$ & $9\left(\mathrm{C}_{4 \mathrm{v}}\right)$ & \multirow[t]{2}{*}{$2 \mathrm{D}$} \\
\hline & Dy2 & 4 & $\begin{array}{l}\text { DMSO-1 } \\
\mathrm{H}_{2} \mathrm{O}-1\end{array}$ & Muffin & $9(\mathrm{Cs})$ & \\
\hline 4 & Dy1 & 3 & $\begin{array}{l}\text { DEF-1 } \\
\mu 2-\mathrm{OH}-1\end{array}$ & $\begin{array}{l}\text { Pentagonal } \\
\text { Pyramidal }\end{array}$ & $6\left(\mathrm{C}_{5 \mathrm{v}}\right)$ & $1 \mathrm{D}$ \\
\hline 5 & Dy1 & 5 & Phen-1 & $\begin{array}{l}\text { Distorted Square } \\
\text { antiprism }\end{array}$ & $8\left(\mathrm{D}_{4 \mathrm{~d}}\right)$ & $1 \mathrm{D}$ \\
\hline 6 & Dy1 & 3 & $\begin{array}{l}\mathrm{Py}-2 \\
\mathrm{NO}_{3}-1\end{array}$ & $\begin{array}{c}\text { Triangular } \\
\text { dodecahedron }\end{array}$ & $8\left(\mathrm{D}_{2 \mathrm{~d}}\right)$ & $1 \mathrm{D}$ \\
\hline
\end{tabular}

